\title{
Liver-First Approach for Synchronous Colorectal Metastases: Analysis of 7360 Patients from the LiverMetSurvey Registry
}

\author{
Felice Giuliante, $\mathrm{MD}^{1}$, Luca Viganò, $\mathrm{MD}, \mathrm{PhD}^{2}$, Agostino M. De Rose, $\mathrm{MD}$, $\mathrm{PhD}^{1}$, Darius F. Mirza, $\mathrm{MD}, \mathrm{PhD}^{3}$, \\ Réal Lapointe, $\mathrm{MD}^{4}$, Gernot Kaiser, $\mathrm{MD}^{5}$, Eduardo Barroso, $\mathrm{MD}^{6}$, Alessandro Ferrero, $\mathrm{MD}^{7}$, \\ Helena Isoniemi, $\mathbf{M D}^{8}$, Santiago Lopez-Ben, $\mathrm{MD}^{9}$, Irinel Popescu, $\mathrm{MD}^{10}$, Jean-Francois Ouellet, $\mathbf{M D}^{11}$, \\ Catherine Hubert, $\mathrm{MD}^{12}$, Jean-Marc Regimbeau, $\mathrm{MD}^{13}$, Jen-Kou Lin, MD ${ }^{14}$, Oleg G. Skipenko, MD ${ }^{15}$, \\ Francesco Ardito, MD, $\mathbf{P h D}^{1}$, and René Adam, $\mathrm{MD}^{16}$
}

${ }^{1}$ Hepatobiliary Surgery Unit, Foundation "Policlinico Universitario A. Gemelli", IRCCS, Catholic University, Rome, Italy; ${ }^{2}$ Division of Hepatobiliary and General Surgery, Department of Surgery, IRCCS - Humanitas Clinical and Research Center, Rozzano, Milan, Italy; ${ }^{3}$ HPB Surgery, University Hospitals Birmingham NHS Foundation Trust, Birmingham, UK; ${ }^{4}$ Hepatopancreatobiliary Surgery and Liver Transplantation Service, Centre Hospitalier de l'Université de Montréal (CHUM), Montreal, QC, Canada; ${ }^{5}$ Department of General, Visceral and Transplantation Surgery, University Hospital of Essen, Essen, Germany; ${ }^{6} \mathrm{HBP}$ and Transplantation Centre, Curry Cabral Hospital, Lisbon Central Hospitals Centre, Lisbon, Portugal; ${ }^{7}$ Department of General and Oncological Surgery, "Umberto I" Mauriziano Hospital, Turin, Italy; ${ }^{8}$ Department of Liver Surgery and Transplantation, Helsinki University, Helsinki, Finland; ${ }^{9}$ Hepatobiliary and Pancreatic Surgery Unit, Department of Surgery, Dr. Josep Trueta Hospital, IdlBGi, Girona, Spain; ${ }^{10}$ Department of Surgery and Transplantation, Fundeni Clinical Institute, Bucharest, Romania; ${ }^{11} \mathrm{CHU}$ de Québec - Université Laval, Quebec City, QC, Canada; ${ }^{12}$ Department of HBP Surgery, Cliniques Universitaires Saint-Luc, Université Catholique de Louvain, Leuven, Belgium; ${ }^{13}$ Department of Oncology and Digestive Surgery, CHU Amiens-Picardie, Amiens, France; ${ }^{14}$ Division of Colon and Rectal Surgery, Department of Surgery, Taipei Veterans General Hospital and School of Medicine, National YangMing University, Taipei, Taiwan; ${ }^{15}$ Research Center of Surgery, Russian Academy of Medical Science, Moscow, Russia; ${ }^{16}$ Department of Surgery, Paul-Brousse Hospital, Assistance Publique Hôpitaux de Paris, Centre Hépato-Biliaire, Villejuif, France

\begin{abstract}
Background. The liver-first approach in patients with synchronous colorectal liver metastases (CRLM) has gained wide consensus but its role is still to be clarified. We aimed to elucidate the outcome of the liver-first approach and to identify patients who benefit at most from this approach.
\end{abstract}

Supplementary Information The online version contains supplementary material available at https://doi.org/10.1245/s10434021-10220-w.

(C) The Author(s) 2021

First Received: 30 December 2020

Accepted: 8 May 2021;

Published Online: 1 July 2021

F. Giuliante, MD

e-mail: felice.giuliante@unicatt.it
Methods. Patients with synchronous CRLM included in the LiverMetSurvey registry between 2000 and 2017 were considered. Three strategies were analyzed, i.e. liver-first approach, colorectal resection followed by liver resection (primary-first), and simultaneous resection, and three groups of patients were analyzed, i.e. solitary metastasis, multiple unilobar CRLM, and multiple bilobar CRLM. In each group, patients from the three strategy groups were matched by propensity score analysis.

Results. Overall, 7360 patients were analyzed: 4415 primary-first, 552 liver-first, and 2393 simultaneous resections. Compared with the other groups, the liver-first group had more rectal tumors $(58.0 \%$ vs. $31.2 \%)$ and higher hepatic tumor burden (more than three CRLMs: $34.8 \%$ vs. $24.0 \%$; size $>50 \mathrm{~mm}: 35.6 \%$ vs. $22.8 \%$; $p<0.001$ ). In patients with solitary and multiple unilobar CRLM, survival was similar regardless of treatment strategy, whereas in patients with multiple bilobar metastases, 
the liver-first approach was an independent positive prognostic factor, both in unmatched patients (3-year survival $65.9 \%$ vs. primary-first $60.4 \%$ : hazard ratio [HR] 1.321 , $p=0.031$; vs. simultaneous resections $54.4 \%$ : HR 1.624 , $p<0.001$ ) and after propensity score matching (vs. primary-first: HR 1.667, $p=0.017$; vs. simultaneous resections: HR 2.278, $p=0.003$ ).

Conclusion. In patients with synchronous CRLM, the surgical strategy should be decided according to the hepatic tumor burden. In the presence of multiple bilobar CRLM, the liver-first approach is associated with longer survival than the alternative approaches and should be evaluated as standard.

Colorectal cancer is the second most common cancer in Europe and the second cause of cancer-related mortality worldwide. ${ }^{1,2}$ At first diagnosis, up to $20 \%$ of patients with colorectal cancer have liver metastases (CRLM) that reflect poor tumor biology and prognosis. ${ }^{3-5}$ Nevertheless, complete resection of both primary tumor and CRLM improves survival expectancy compared with systemic therapies. ${ }^{6-10}$

The management of patients with synchronous CRLM requires complex multidisciplinary evaluation as long as colorectal surgery, hepatic surgery, and chemotherapy are combined with appropriate timing. Traditional approaches schedule either simultaneous colorectal and hepatic resection or colorectal resection followed by liver resection (primary tumor-first approach), administering chemotherapy in the perioperative period. In 2006, Mentha et al. proposed a reverse strategy (liver-first approach), scheduling first liver resection and then primary tumor resection to prioritize the removal of the most prognostically relevant disease (liver metastases) and to ease the inclusion of radiotherapy for locally advanced rectal tumors. ${ }^{11}$ Even if appealing, the liver-first approach failed to demonstrate its superiority over the alternatives, mainly because of different patient selection in a determined single institution. ${ }^{12-22}$ To date, the choice of treatment strategy of synchronous CRLM relies on a case-by-case evaluation by every single multidisciplinary expert team rather than on robust evidence or proven benefits.

The present study aims to elucidate the role of reverse strategy in the management of patients with synchronous CRLM, analyzing the impact of treatment strategy in patients at different tumor burden. Thanks to the analysis of a large multicenter international cohort of patients, we have solid expectancy to identify patients who benefit most from a reverse strategy.

\section{METHODS}

All patients with synchronous CRLM included in the LiverMetSurvey registry (www. livermetsurvey-arcad.org) undergoing liver surgery between January 2000 and December 2017 were reviewed. The characteristics of the LiverMetSurvey registry have been previously reported. ${ }^{23-25}$ Patients were divided into three groups according to the adopted surgical strategy: (1) colorectal resection followed by liver resection (classical approach, primaryfirst group); (2) liver resection followed by colorectal resection (reverse strategy, liver-first group); (3) simultaneous colorectal and hepatic resection (simultaneous group). Exclusion criteria were unresected patients, missing or incomplete data regarding surgical procedures, delay between primary tumor and liver resection of $>1$ year in the primary-first group, and two-stage hepatectomy.

\section{Study Design}

The liver-first group was compared with the other two approaches in terms of short- and long-term outcomes. Furthermore, we compared the outcome of the three approaches in three subgroups of patients, classified according to their hepatic tumor burden: patients with solitary metastasis, patients with multiple unilobar metastases, and patients with multiple bilobar metastases. Considering short-term outcome analysis, in the primaryfirst and liver-first groups we considered only complications related to liver resection because complications related to colorectal resection are not reported in the registry. We analyzed 90-day mortality, overall morbidity, hepatic complication (i.e. liver failure, bile leak, infected perihepatic collection, bleeding from the cut surface, or ascites), and infectious complication rates. Considering survival analysis, in the three subgroups (solitary, multiple unilobar, and multiple bilobar CRLM) we compared the three approaches before and after propensity score matching of patients (see details in the Statistical Analysis section). Of note, the registry includes only patients operated on for CRLM. Consequently, even if all data regarding patients' history are collected, LiverMetSurvey data do not allow an intention-to-treat analysis to be performed of patients with synchronous CRLM.

\section{Statistical Analysis}

Categorical variables were compared using the Chisquare test or Fisher's exact test, as appropriate, whereas continuous variables were compared using parametric (unpaired $t$ test) or non-parametric (Mann-Whitney $U$-test) tests, as appropriate. The Kaplan-Meier method was used to estimate survival probabilities, which were compared 
using the log-rank test. Overall survival (OS) and recurrence-free survival (RFS) were calculated from the date of liver surgery. Furthermore, we calculated OS from the date of diagnosis of CRLM, and OS from the date of completion of surgical strategy, i.e. from the date of liver surgery in the primary-first and simultaneous groups and from the date of colorectal surgery in the liver-first group. Cox proportional hazards regression models were used to evaluate the association of the relevant clinicopathological factors with prognosis. Variables with a $p$ value $<0.10$ at univariate analysis and those associated with outcome in the literature were included in the multivariable analysis. Carcinoembryonic antigen (CEA) values and RAS status were not included in the analysis because the former was missing in a high proportion of patients (34\%) and the latter was not reported in the registry.

When we performed the survival analysis of the three subgroups of patients (solitary CRLM, multiple unilobar CRLM, and multiple bilobar CRLM), we used a propensity score analysis to overcome biases owing to the different distribution of covariates among patients in the three approach groups. In every subgroup, two separate propensity score analyses were performed: the first to match (1:2) patients in the liver-first group with patients in the primary-first group; and the second to match (1:1) patients in the liver-first group with patients in the simultaneous group. Separate multivariable logistic regression models were performed (one per each propensity score analysis) to predict the probability of each patient being submitted to a surgical approach on the basis of the following covariates: age, year of resection, primary tumor site, $\mathrm{N}$ status of the primary tumor, number of CRLMs, size of CRLM, extrahepatic disease, and preoperative chemotherapy. The nearest-neighbor matching method was used. Matching to 5 decimal points was performed, followed by 4-, 3-, and 2-point matching. Cases whose propensity score deviated $>0.01$ were considered unmatched and were hence excluded.

All analyses were carried out using SPSS version 25 (IBM Corporation, Armonk, NY, USA) and STATA version 14 (StataCorp LLC, Cary, NC, USA).

\section{RESULTS}

Overall, 12,744 patients with synchronous CRLM who were included in the LiverMetSurvey registry were considered. A total of 5384 patients were excluded (908 unresected patients, 2835 patients with missing or incomplete data regarding surgical procedures, 909 patients with a delay between primary tumor and liver resection $>1$ year, and 732 two-stage hepatectomies), resulting in 7360 patients being analyzed: 4415 patients $(60.0 \%)$ in the primary-first group, $552(7.5 \%)$ patients in the liver-first group, and 2393 (32.5\%) patients in the simultaneous group (electronic supplementary Fig. 1).

The liver-first approach increased over time, from $2.4 \%$ before 2007 to $5.9 \%$ in the period 2007-2011, and to $12.6 \%$ later on $(p<0.001)$, while the primary-first approach decreased $(68.3 \%, 58.2 \%$, and $57.3 \%$, respectively; $p<0.001)$ and the simultaneous group remained stable $(\approx 30 \%)$. During the study period, the number of resected metastases did not have a significant increase (three or more nodules: $23.9 \%$ before $2007,24.8 \%$ in the period 2007-2011, and 26.1\% after 2011), while metastasis size decreased $(>50 \mathrm{~mm}: 27.8 \%, 23.1$, and $22.2 \%$, respectively; $p=0.001$ ). The proportion of patients undergoing preoperative chemotherapy progressively increased $(42.1 \%, 50.9 \%$, and $54.1 \%$, respectively; $p<0.001)$.

The three groups of patients had major differences. The liver-first group included more patients with rectal tumors $(58.0 \%$ vs. $31.2 \%$ in the other groups; $p<0.001)$ and fewer patients with right/transverse colon cancers $(12.4 \%$ vs. $26.4 \% ; p<0.001)$. Among patients with rectal tumors, patients undergoing a reverse strategy more often had pelvic radiotherapy $(56.6 \%$ vs. $33.3 \% ; p<0.001)$. The liver-first group had the highest tumor burden in terms of CRLM number (more than three in $34.8 \%$ vs. $24.0 \%$; $p<0.001$ ), size ( $>50 \mathrm{~mm}$ in $35.6 \%$ vs. $22.8 \% ; p<0.001$ ), and bilobar distribution $(51.5 \%$ vs. $38.8 \%, p<0.001)$. In comparison with the primary-first strategy, the reverse strategy was not only associated with more preoperative chemotherapy (75.7\% vs. $57.0 \% ; p<0.001)$ but also with a shorter treatment (more than six cycles $57.2 \%$ vs. $62.78 \% ; p=0.044$ ) and a shorter interval between the two surgical procedures (colorectal and liver resection, mean 92 vs. 162 days; $p<0.001)$. Table 1 summarizes the patient characteristics.

In the whole series, the liver-first group had 90-day mortality, overall morbidity, and hepatic morbidity rates similar to the other groups. Staged resections (liver-first and primary-first groups) had a lower infectious complication rate than the simultaneous group $(11.4 \%$ vs. $18.6 \%$; $p<0.001)$. The same results were observed considering patients with solitary or multiple unilobar metastases. Considering patients with multiple bilobar metastases, the liver-first group had 90-day mortality, overall morbidity, and infectious morbidity rates similar to the primary-first group $(2.3 \%$ vs. $2.0 \%, 31.1 \%$ vs. $30.4 \%$, and $12.0 \%$ vs. $11.8 \%$, respectively), but lower than the simultaneous group (vs. $5.1 \%, p=0.052$; vs. $39.9 \%, p=0.016$; vs. $20.1 \%, p=0.006$, respectively). Hepatic complication rates were similar among the three groups. Considering patients who require a major hepatectomy, independently of the tumor burden, mortality was lower in the liver-first 
TABLE 1 Patient characteristics according to the surgical strategy

\begin{tabular}{|c|c|c|c|c|c|}
\hline Variable & $\begin{array}{l}\text { Liver-first } \\
(n=552)\end{array}$ & $\begin{array}{l}\text { Primary-first } \\
(n=4415)\end{array}$ & $\begin{array}{l}p \text { value } \\
\text { Primary-first } \\
\quad \text { versus liver-first }\end{array}$ & $\begin{array}{l}\text { Simultaneous } \\
(n=2393)\end{array}$ & $\begin{array}{l}p \text { value } \\
\text { Simultaneous } \\
\quad \text { versus liver-first }\end{array}$ \\
\hline Sex (female) & $205(37.1)$ & $1794(40.6)$ & 0.114 & $985(41.2)$ & 0.082 \\
\hline Age $>70$ years & $111(20.1)$ & $896(20.3)$ & 0.918 & $658(27.5)$ & $<0.001$ \\
\hline \multicolumn{6}{|l|}{ Primary cancer } \\
\hline \multicolumn{6}{|l|}{ Tumor site } \\
\hline Right/transverse colon & $68 / 547(12.4)$ & $993 / 4318(23.0)$ & $<0.001$ & 778/2383 (32.7) & $<0.001$ \\
\hline Left colon & $162 / 547(29.6)$ & $1994 / 4318(46.2)$ & & $848 / 2383(35.6)$ & \\
\hline Rectum & $317 / 547(58.0)$ & $1331 / 4318(30.8)$ & & $757 / 2383(31.8)$ & \\
\hline $\mathrm{T}$ stage $(\mathrm{T} 3-4)$ & 416/427 (97.4) & $3795 / 3834(99.0)$ & 0.005 & 2078/2106 (98.7) & 0.056 \\
\hline LN metastases (N1) & $325 / 499(65.1)$ & $2971 / 4080(72.8)$ & $<0.001$ & $1553 / 2232(69.6)$ & 0.053 \\
\hline Radiotherapy if rectal cancer & $172 / 304(56.6)$ & $389 / 1250(31.1)$ & $<0.001$ & $274 / 739(37.1)$ & $<0.001$ \\
\hline \multicolumn{6}{|l|}{ Liver metastases } \\
\hline Single metastasis & $144 / 532(27.1)$ & $1501 / 4198(35.8)$ & $<0.001$ & $1118 / 2308(48.4)$ & $<0.001$ \\
\hline Metastases $>3$ & $185 / 532(34.8)$ & $1129 / 4198(26.9)$ & $<0.001$ & 430/2308 (18.6) & $<0.001$ \\
\hline Metastasis $>5 \mathrm{~cm}$ & $173 / 486(35.6)$ & $881 / 3827(23.0)$ & $<0.001$ & $462 / 2069(22.3)$ & $<0.001$ \\
\hline Bilateral metastases & $281 / 546(51.5)$ & $1837 / 4345(42.3)$ & $<0.001$ & $762 / 2354(32.4)$ & $<0.001$ \\
\hline $\mathrm{R} 1$ resection $^{\mathrm{a}}$ & $97 / 463(21)$ & $592 / 3585(16.5)$ & 0.017 & 193/1715 (11.3) & $<0.001$ \\
\hline Extrahepatic disease & $35(6.3)$ & $187(4.2)$ & 0.024 & $160(6.7)$ & 0.768 \\
\hline Lung metastases & $28(5.1)$ & $123(2.8)$ & 0.003 & $73(3.1)$ & 0.019 \\
\hline LN metastases & $3(0.5)$ & $20(0.5)$ & 0.768 & $23(1.0)$ & 0.344 \\
\hline Hepatic pedicle LNs & - & 6 & 1.000 & 6 & 0.601 \\
\hline $\mathrm{CEA}>200 \mathrm{ng} / \mathrm{mL}$ & $19 / 383(5.0)$ & $128 / 2912(4.4)$ & 0.614 & $104 / 1568(6.6)$ & 0.227 \\
\hline \multicolumn{6}{|l|}{ Chemotherapy data } \\
\hline Neoadjuvant chemotherapy & $418 / 552(75.7)$ & $2516 / 4413(57.0)$ & $<0.001$ & 757/2393 (31.6) & $<0.001$ \\
\hline Chemotherapy lines $>1$ & $52 / 418(12.4)$ & $264 / 2516(10.5)$ & 0.234 & $104 / 755(13.8)$ & 0.519 \\
\hline Chemotherapy cycles $>6$ & $210 / 367(57.2)$ & $1380 / 2200(62.7)$ & 0.044 & $369 / 647(57.0)$ & 0.954 \\
\hline \multicolumn{6}{|l|}{ Response to chemotherapy } \\
\hline $\mathrm{CR}$ & $10 / 388(2.6)$ & $72 / 2268(3.2)$ & 0.004 & 26/704 (3.7) & 0.382 \\
\hline PR & $305 / 388(78.6)$ & $1578 / 2268(69.6)$ & & $522 / 704(74.2)$ & \\
\hline SD & $60 / 388(15.5)$ & $500 / 2268(22.1)$ & & $125 / 704(17.8)$ & \\
\hline PD & $13 / 388(3.4)$ & $118 / 2268(5.2)$ & & $31 / 704(4.4)$ & \\
\hline \multicolumn{6}{|l|}{ Resection details } \\
\hline \multicolumn{6}{|l|}{ Type of resection } \\
\hline Anatomic & $215 / 541(39.7)$ & $1806 / 4281(42.2)$ & 0.008 & $720 / 2308(31.2)$ & $<0.001$ \\
\hline Anatomic + non-anatomic & $183 / 541(33.8)$ & $1180 / 4281(27.6)$ & & $345 / 2308(15.0)$ & \\
\hline Non-anatomic & $143 / 541(26.4)$ & $1295 / 4281(30.3)$ & & $1243 / 2308(53.9)$ & \\
\hline Major hepatectomy & $145 / 357(40.6)$ & $1219 / 3202(38.1)$ & 0.348 & 290/1973 (14.7) & $<0.001$ \\
\hline Associated intraoperative thermal ablation & $77 / 552(14.0)$ & $524 / 4413(11.9)$ & 0.159 & $165 / 2392(6.9)$ & $<0.001$ \\
\hline
\end{tabular}

Data are expressed as $n / N(\%)$ unless otherwise specified

$L N$ lymph node, $C E A$ carcinoembryonic antigen, $C R$ complete response, $P R$ partial response, $S D$ stable disease, $P D$ progression of disease ${ }^{\mathrm{a}} \mathrm{R} 1$ resection refers to the surgical margin of liver resection

group (4.8\%) and primary-first group (2.9\%) than in the simultaneous group $(8.1 \% ; p<0.001)$. Short-term outcomes are detailed in electronic supplementary Table 1.
Survival Analysis

Whole Series After a median follow-up of 37 months, 5 -year OS after liver resection was $46.6 \%$ (median OS 53.0 months, median OS since diagnosis of CRLM 59.3 


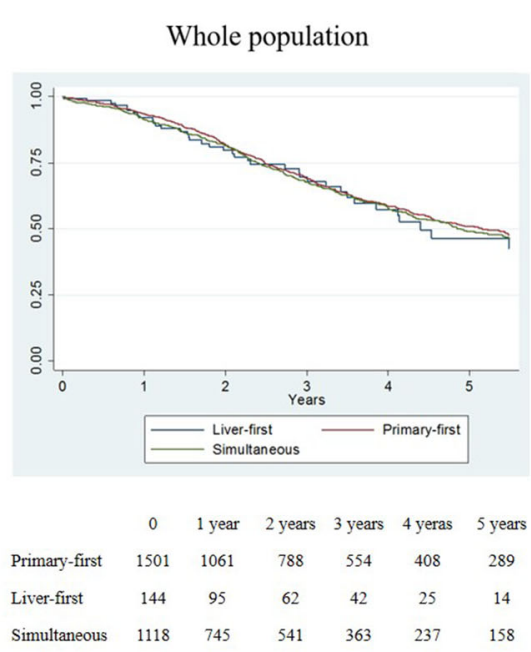

Liver-first vs. Primary-first after PSM
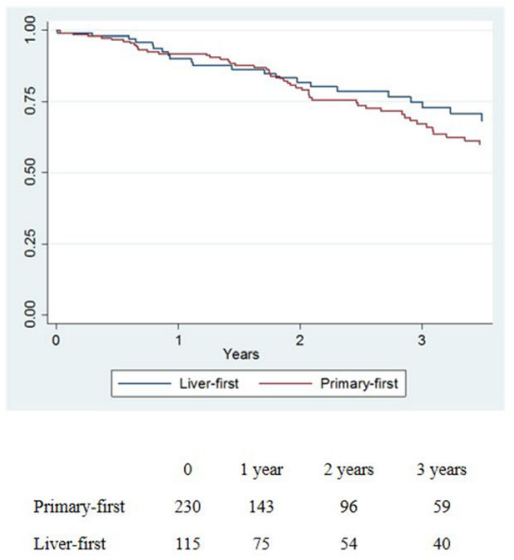

Liver-first vs. Simultaneous after PSM
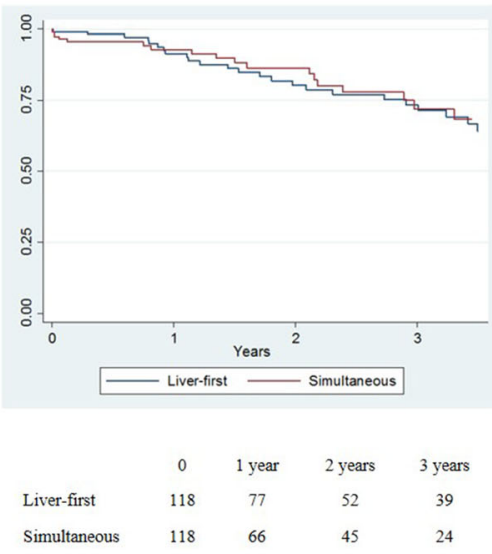

FIG. 1 Overall survival in patients with solitary metastasis according to the treatment strategy. a Whole population, b liver-first group versus primary-first group after PSM, c liver-first group versus simultaneous group after PSM. PSM propensity score matching

months). The liver-first approach had the longest OS (at 5 years $51.4 \%$; median OS 65.4 months), followed by the primary-first approach $(47.1 \%, 53.9$ months; $p=0.213)$ and the simultaneous group $(44.8 \%, 51.0$ months, $p=0.014$ vs. the liver-first group) (electronic supplementary Fig. 2). Median OS since diagnosis of CRLM was 72.4, 61.0, and 53.3 months in the liver-first, primary-first, and simultaneous groups, respectively. Considering RFS, recurrence data were available for 7084 patients. The liver-first group had the lowest recurrence rate $(36.8 \%$ [200/544] vs. 38.7\% [890/2302] in the simultaneous group, and $45.6 \%$ [1931/4238] in the colon-first group; $p<0.001)$. The liver-first group had RFS similar to the simultaneous group (liver-first group: 5-year RFS $37.5 \%$ and median RFS 28.2 months; simultaneous group: 5-year RFS $37.9 \%$ and median RFS 30.5 months), higher than the primary-first group (5-year RFS $34.6 \%$ and median RFS 23.9 months, $p=0.043$ ).

At multivariable analysis, OS after liver resection of the liver-first group was better than that of the simultaneous group (hazard ratio [HR] 1.298 for the latter group; $p=0.036$ ), and similar to that of the primary-first group (HR 1.212; $p=0.100$ ) (electronic supplementary Table 2).

Solitary Metastasis In patients with solitary metastasis, OS was not associated with treatment strategy (liver-first group vs. primary-first group: 3 -year OS $69.6 \%$ vs. $69.1 \%$, HR $0.932, p=0.672$; and liver-first group vs. simultaneous group: 3-year OS $69.6 \%$ vs. $67.8 \%$, HR $0.979, p=0.898$ ) (Fig. 1a). After propensity score matching, we compared (1) 115 patients in the liver-first group versus 230 patients in the primary-first group; and (2) 118 patients in the liverfirst group versus 118 patients in the simultaneous group. The characteristics of the groups were similar after matching (Table 2). The liver-first group had survival similar to both the primary-first group (3-year OS $74.9 \%$ vs. $67.1 \%$, HR $0.842, p=0.445$ ) and the simultaneous group $(73.3 \%$ vs. $71.9 \%$, HR $1.218, p=0.486)$ (Fig. 1b, c). Multivariable analyses in matched groups confirmed no association between treatment strategy and OS after liver resection. Similarly, there was no association between treatment strategy and OS since CRLM diagnosis, OS after completion of treatment strategy, and RFS (data not shown).

Multiple Unilobar Metastases In patients with multiple unilobar metastases, the liver-first group had OS similar to the primary-first group (at 3 years: $72.2 \%$ vs. $65.6 \%$, HR $1.164, p=0.416)$, but longer than the simultaneous group (58.1\%, HR 1.414, $p=0.076$ ) (Fig. 2a). After propensity score matching, we compared (1) 84 patients in the liverfirst group versus 168 patients in the primary-first group; and (2) 73 patients in the liver-first group versus 73 patients in the simultaneous group. The characteristics of the groups were similar after matching (Table 2). The liver-first and primary-first groups had similar survival (3-year OS 78.0\% vs. $70.4 \% ; p=0.551$ ), while the liver-first group had slightly longer survival than the simultaneous group (73.2\% vs. $59.7 \% ; p=0.239$ ) (Fig. $2 \mathrm{~b}, \mathrm{c}$ ). Multivariable analyses in matched groups showed no association between treatment strategy and OS after liver resection. Similarly, there was no evidence of an association between treatment strategy and OS since CRLM diagnosis, OS after completion of treatment strategy, and RFS (data not shown).

Multiple Bilobar Metastases In patients with multiple bilobar metastases, the liver-first group had longer survival 
TABLE 2 Patient characteristics after propensity score matching

\begin{tabular}{|c|c|c|c|c|c|c|}
\hline & Liver-first & Primary-first & $\begin{array}{l}p \text { value } \\
\text { Liver-first versus } \\
\text { primary-first }\end{array}$ & Liver-first & Simultaneous & $\begin{array}{l}p \text { value } \\
\text { Liver-first versus } \\
\quad \text { simultaneous }\end{array}$ \\
\hline \multicolumn{7}{|l|}{ Single metastasis } \\
\hline & $\mathrm{n}=115$ & $\mathrm{n}=230$ & & $\mathrm{n}=118$ & $\mathrm{n}=118$ & \\
\hline Age $>70$ years & $25(21.7)$ & $44(19.1)$ & 0.568 & $29(24.6)$ & $30(25.4)$ & 0.881 \\
\hline \multicolumn{7}{|l|}{ Year of surgery } \\
\hline 2000-2006 & $10(8.7)$ & $19(8.3)$ & 0.990 & $10(8.5)$ & $10(8.5)$ & 0.990 \\
\hline $2007-2011$ & $37(32.2)$ & $74(32.2)$ & & $37(31.4)$ & $36(30.5)$ & \\
\hline 2012-2017 & $68(59.1)$ & $137(59.6)$ & & $71(60.2)$ & $72(61.0)$ & \\
\hline \multicolumn{7}{|l|}{ Tumor site } \\
\hline Right/transverse colon & $16(13.9)$ & $31(13.5)$ & 0.992 & 16 (13.6) & 16 (13.6) & 0.902 \\
\hline Left colon & 34 (29.6) & $69(30.0)$ & & $33(28.0)$ & $30(25.4)$ & \\
\hline Rectum & $65(56.5)$ & $130(56.5)$ & & $69(58.5)$ & $72(61.0)$ & \\
\hline $\mathrm{N}+$ primary tumor & $76(66.1)$ & $153(66.5)$ & 0.936 & $78(66.1)$ & $80(67.8)$ & 0.782 \\
\hline Metastases diameter $>50 \mathrm{~mm}$ & $38(33.0)$ & $74(32.2)$ & 0.871 & $39(33.1)$ & $36(30.5)$ & 0.675 \\
\hline Extrahepatic disease & $2(1.7)$ & $4(1.7)$ & 1.000 & $2(1.7)$ & $2(1.7)$ & 1.000 \\
\hline Preoperative chemotherapy & $69(60.0)$ & $135(58.7)$ & 0.816 & $65(55.1)$ & $64(54.2)$ & 0.896 \\
\hline \multicolumn{7}{|l|}{ Unilobar multiple metastases } \\
\hline & $\mathrm{n}=84$ & $\mathrm{n}=168$ & & $\mathrm{n}=73$ & $\mathrm{n}=73$ & \\
\hline Age $>70$ years & $14(16.7)$ & $19(11.3)$ & 0.235 & $13(17.8)$ & $10(13.7)$ & 0.496 \\
\hline \multicolumn{7}{|l|}{ Year of surgery } \\
\hline 2000-2006 & $4(4.8)$ & $9(5.4)$ & 0.952 & $4(5.5)$ & $5(6.8)$ & 0.862 \\
\hline $2007-2011$ & $34(40.5)$ & $65(38.7)$ & & $31(42.5)$ & $33(45.2)$ & \\
\hline 2012-2017 & $46(54.8)$ & $94(56.0)$ & & $38(52.1)$ & $35(47.9)$ & \\
\hline \multicolumn{7}{|l|}{ Tumor site } \\
\hline Right/transverse colon & $13(15.5)$ & $28(16.7)$ & 0.935 & $13(17.8)$ & $11(15.1)$ & 0.827 \\
\hline Left colon & $25(29.8)$ & $52(31.0)$ & & $21(28.8)$ & $24(32.9)$ & \\
\hline Rectum & $46(54.8)$ & $88(52.4)$ & & $39(53.4)$ & $38(52.1)$ & \\
\hline $\mathrm{N}+$ primary tumor & $53(63.1)$ & $104(61.9)$ & 0.854 & 45 (61.6) & $46(63.0)$ & 0.864 \\
\hline Number of metastases $>3$ & $15(17.9)$ & $27(16.1)$ & 0.720 & $15(20.5)$ & $15(20.5)$ & 1.000 \\
\hline Number of metastases [mean (range)] & $2.8(2-9)$ & $2.7(2-7)$ & 0.297 & $2.9(2-9)$ & $2.9(2-8)$ & 0.952 \\
\hline Metastases diameter $>50 \mathrm{~mm}$ & $22(26.2)$ & $45(26.8)$ & 0.920 & $18(24.7)$ & $21(28.8)$ & 0.575 \\
\hline Extrahepatic disease & - & - & 1.000 & $1(1.4)$ & $1(1.4)$ & 1.000 \\
\hline Preoperative chemotherapy & $60(71.4)$ & $120(71.4)$ & 1.000 & $49(67.1)$ & $50(68.5)$ & 0.859 \\
\hline \multicolumn{7}{|l|}{ Bilobar multiple metastases } \\
\hline & $\mathrm{n}=163$ & $\mathrm{n}=326$ & & $\mathrm{n}=135$ & $\mathrm{n}=135$ & \\
\hline Age $>70$ years & $33(20.2)$ & $66(20.2)$ & 1.000 & $25(18.5)$ & $23(17.0)$ & 0.750 \\
\hline \multicolumn{7}{|l|}{ Year of surgery } \\
\hline 2000-2006 & $7(4.3)$ & $11(3.4)$ & 0.737 & $7(5.2)$ & $7(5.2)$ & 1.000 \\
\hline $2007-2011$ & $57(35.0)$ & $124(38.0)$ & & $55(40.7)$ & $55(40.7)$ & \\
\hline $2012-2017$ & 99 (60.7) & $191(58.6)$ & & $73(54.1)$ & $73(54.1)$ & \\
\hline \multicolumn{7}{|l|}{ Tumor site } \\
\hline Right/transverse colon & $25(15.3)$ & $46(14.1)$ & 0.924 & $23(17.0)$ & $24(17.8)$ & 0.984 \\
\hline Left colon & $58(35.6)$ & $120(36.8)$ & & $45(33.3)$ & $44(32.6)$ & \\
\hline Rectum & $80(49.1)$ & $160(49.1)$ & & 67 (49.6) & $67(49.6)$ & \\
\hline $\mathrm{N}+$ primary tumor & $113(66.0)$ & $215(69.3)$ & 0.454 & $92(68.1)$ & $93(68.9)$ & 0.896 \\
\hline Number of metastases $>3$ & $96(58.9)$ & $192(58.9)$ & 1.000 & $76(56.3)$ & $77(57.0)$ & 0.902 \\
\hline Number of metastases [mean (range)] & $5.3(2-25)$ & $5.0(2-36)$ & 0.309 & $5.2(2-25)$ & $4.6(2-20)$ & 0.217 \\
\hline Metastases diameter $>50 \mathrm{~mm}$ & $51(31.3)$ & $109(33.4)$ & 0.633 & $46(34.1)$ & $44(32.6)$ & 0.796 \\
\hline Extrahepatic disease & $2(1.2)$ & $4(1.2)$ & 1.000 & $2(1.5)$ & $2(1.5)$ & 1.000 \\
\hline Preoperative chemotherapy & $141(86.5)$ & $275(84.4)$ & 0.530 & $113(83.7)$ & $114(84.4)$ & 0.868 \\
\hline
\end{tabular}




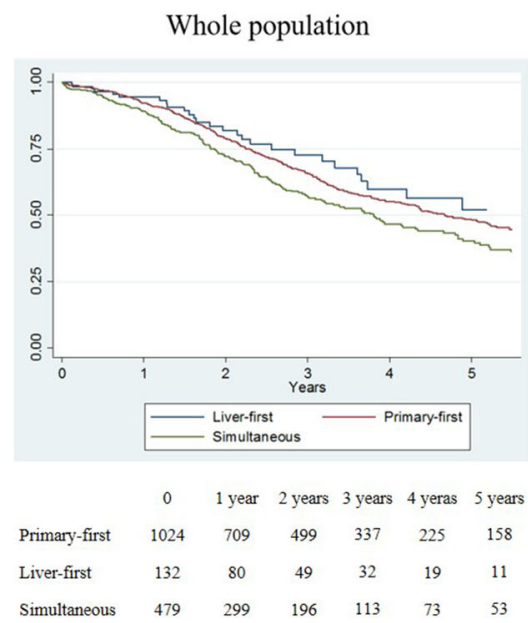

Liver-first vs. Primary-first after PSM
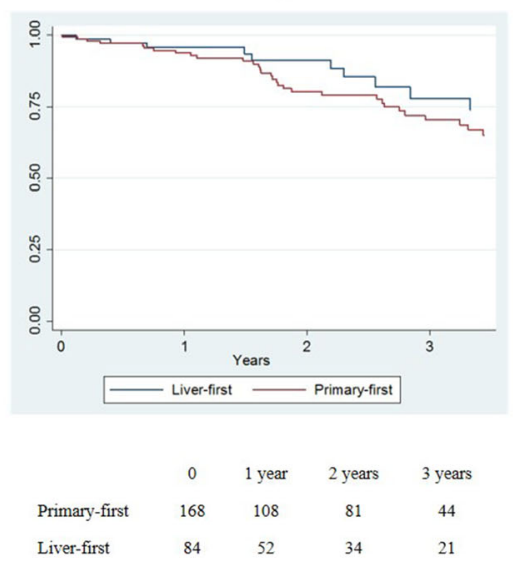

Liver-first vs. Simultaneous after PSM

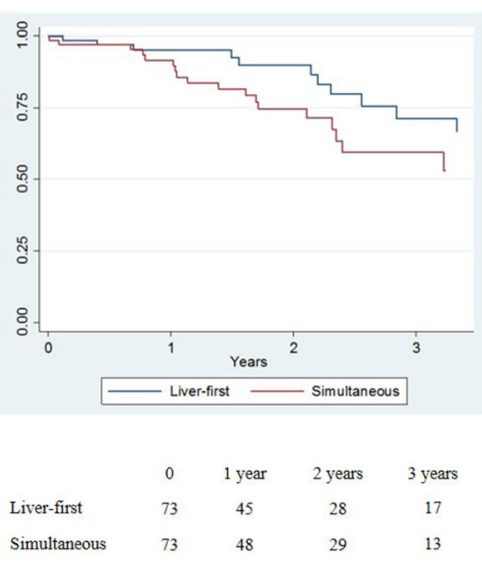

FIG. 2 Overall survival in patients with multiple unilobar metastases according to the treatment strategy. a Whole population, b liver-first group versus primary-first group after PSM, c liver-first group versus simultaneous group after PSM. PSM propensity score matching
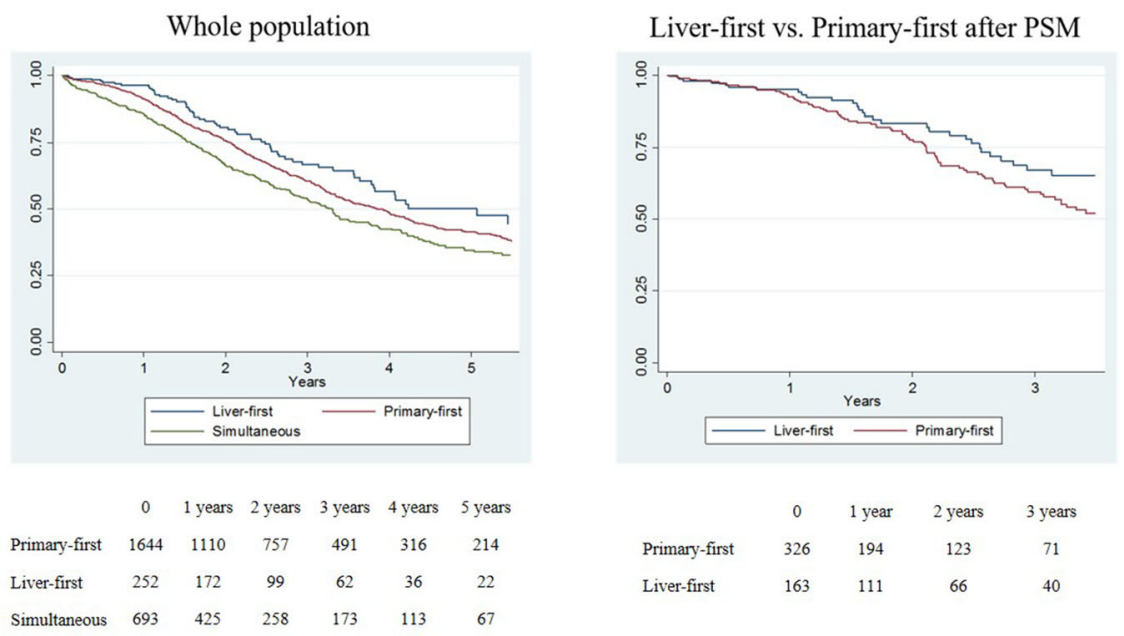

Liver-first vs. Simultaneous after PSM

FIG. 3 Overall survival in patients with multiple bilobar metastases according to the treatment strategy. a Whole population, b liver-first group versus primary-first group after PSM, c liver-first group verss simultaneous group after PSM. PSM propensity score matching

than both the primary-first group (at 3 years: $65.9 \%$ vs. $60.4 \%$, HR 1.321, $p=0.031$ ) and the simultaneous group (54.4\%, HR 1.624, $p<0.001$ ) (Fig. 3a). After propensity score matching, we compared (1) 163 patients in the liverfirst group versus 326 patients in the primary-first group; and (2) 135 patients in the liver-first group versus 135 patients in the simultaneous group. The characteristics of the groups were similar after matching (Table 2). In both analyses, the liver-first group had longer OS after liver resection (vs. the primary-first group: 3-year OS $67.1 \%$ vs. $59.6 \%, p=0.064$; vs. the simultaneous group: $68.5 \%$ vs. $50.3 \%, p=0.017$ ) (Fig. 3b, c). Multivariable analyses confirmed the liver-first approach as an independent positive prognostic factor in comparison with both the primary-first approach (HR 1.667; $p=0.017)$ and the simultaneous approach (HR 2.278; $p=0.003$ ) (Table 3). At multivariable analysis, the liver-first approach was associated with longer RFS than the primary-first approach (at 3 years: $37.4 \%$ vs. $26.2 \%$, HR $1.440, p=0.005$ ). The recurrence rate was lower in the liver-first group (39.8\% [64/161] vs. $49.5 \%$ [160/323]), but recurrence site was similar between the two groups (electronic supplementary Table 3). The liver-first group had borderline significantly better RFS than the simultaneous group (40.7\% vs. $34.3 \%$, HR $1.335, p=0.050)$. At multivariable analysis, the liverfirst approach was also associated with longer OS since CRLM diagnosis (vs. primary-first approach: HR 1.656, $p=0.020 ; \quad$ vs. simultaneous resection: HR 2.248, $p=0.003$ ), and OS after completion of the treatment 
TABLE 3 Multivariable analysis of predictive factors of overall survival in patients with multiple bilobar metastases after propensity score matching (simultaneous vs. staged groups)

\begin{tabular}{|c|c|c|}
\hline Parameter & $p$-Value & HR $(95 \% \mathrm{CI})$ \\
\hline \multicolumn{3}{|l|}{ Liver-first vs. primary-first } \\
\hline \multicolumn{3}{|l|}{ Surgical strategy } \\
\hline Liver-first vs. primary-first & 0.017 & $1.667(1.094-2.545)$ \\
\hline \multicolumn{3}{|l|}{ Age } \\
\hline$>70$ vs. $\leq 70$ years & 0.733 & $1.092(0.657-1.817)$ \\
\hline \multicolumn{3}{|l|}{ Year of resection } \\
\hline $2000-2006$ & & 1 \\
\hline $2007-2011$ & 0.558 & $0.806(0.392-1.658)$ \\
\hline 2012-2017 & 0.344 & $0.686(0.314-1.498)$ \\
\hline \multicolumn{3}{|l|}{ Primary tumor site } \\
\hline Left colon & & 1 \\
\hline Right/transverse colon & 0.483 & $0.802(0.434-1.483)$ \\
\hline Rectum & 0.532 & $1.146(0.747-1.759)$ \\
\hline \multicolumn{3}{|l|}{$\mathrm{N}$ status primary tumor } \\
\hline $\mathrm{N}+$ vs. N0 & 0.283 & $1.266(0.823-1.948)$ \\
\hline \multicolumn{3}{|l|}{ Number of metastases $>3$} \\
\hline Y vs. N & 0.900 & $0.975(0.656-1.448)$ \\
\hline \multicolumn{3}{|l|}{ Metastases size $>50 \mathrm{~mm}$} \\
\hline Y vs. N & 0.533 & $0.876(0.576-1.329)$ \\
\hline \multicolumn{3}{|l|}{ Preoperative chemotherapy } \\
\hline Y vs. N & 0.594 & $0.861(0.497-1.492)$ \\
\hline \multicolumn{3}{|l|}{ Complete resection $^{\mathrm{a}}$} \\
\hline R1 vs. R0 & 0.020 & $1.639(1.080-2.488)$ \\
\hline \multicolumn{3}{|c|}{ Associated intraoperative thermal ablation } \\
\hline Y vs. N & 0.396 & $1.206(0.783-1.857)$ \\
\hline
\end{tabular}

Liver-first vs. simultaneous

Surgical strategy

Liver-first vs. simultaneous

0.003

$2.278(1.319-3.937)$

Age

$>70$ vs. $\leq 70$ years

0.703

Year of resection

2000-2006

2007-2011

2012-2017

Primary tumor site

Left colon

Right/transverse colon

Rectum

$\mathrm{N}$ status primary tumor

$\mathrm{N}+$ vs. N0

Number of metastases $>3$

$\mathrm{Y}$ vs. $\mathrm{N}$

Metastases size $>50 \mathrm{~mm}$

Y vs. N

Preoperative chemotherapy

Y vs. N

Complete resection $^{\mathrm{a}}$

R1 vs. R0

Associated intraoperative thermal ablation

Y vs. N strategy (vs. primary-first approach: HR 1.561, $p=0.040$; vs. simultaneous resection: HR 1.946, $p=0.015$ ).

\section{DISCUSSION}

The liver-first approach was initially proposed to include radiotherapy in locally advanced rectal tumors with hepatic metastase ${ }^{11}$ but has generated much interest and obtained good diffusion among patients with synchronous CRLM because of its prioritization to liver disease. Some recent population-based analyses reported its application in up to $20-40 \%$ of patients. ${ }^{17,18,26}$ Nevertheless, the evaluation of its results still relies on a few studies collecting a limited number of cases. ${ }^{19}$ The snapshot from the LiverMetSurvey registry confirmed that the reverse strategy has been more and more applied since its proposal, passing from $2 \%$ before 2007 to $13 \%$ in the most recent years. This gave us the possibility of analyzing a large series including more than 550 patients. The liver-first approach was preferentially applied to patients with rectal tumors and high liver tumor burden (one-quarter of cases in the most recent period). We confirmed the expected benefits from reverse strategy: shorter chemotherapy before liver resection with an excellent response rate, and higher inclusion of pelvic radiotherapy. Nevertheless, the role of the liver-first approach in synchronous CRLM is still to be elucidated and its oncologic superiority over the other strategies is still to be proven.

Several studies focused on the short-term results of the three approaches, most comparing simultaneous and staged procedures with controversial results. ${ }^{27-32}$ The comparison between the primary-first and liver-first approaches did not generate much interest because, as expected, the two had similar outcomes through all studies. ${ }^{12,13,15-17,21}$ In the present series, the three approaches had equivalent results for low-complexity resections, while the simultaneous group had worse outcomes than staged procedures for major hepatectomies and resections of multiple bilobar metastases. Our results are coherent with those recently published by Shubert et al. based on a large US database $(n=43,408){ }^{30}$ They demonstrated that the operative risks of simultaneous resections vary incrementally with the complexity of both hepatectomy and colorectal resection. Even if our picture is incomplete because we lack data about the morbidity of primary tumor resection in staged procedures, we believe that the increased mortality risk of the simultaneous approach in complex procedures is a major point.

Data regarding the long-term outcome are scarce. In 2012, the Geneva group analyzed the LiverMetSurvey registry and demonstrated non-inferiority, but not superiority, of the liver-first approach versus the primary-first

$H R$ hazard ratio, $C I$ confidence intervals, $Y$ yes, $N$ no

${ }^{\mathrm{a}} \mathrm{R} 0 / \mathrm{R} 1$ resection refers to the surgical margin of liver resection 
approach. $^{15}$ Other papers confirmed these data, ${ }^{12,13,15-22,33,34}$ and, to date, no studies have reported a survival advantage of the liver-first approach over the other approaches. Coherently, the EGOSLIM (Expert Group on OncoSurgery management of LIver Metastases) group, ${ }^{6}$ stated that simultaneous resection, when feasible without increasing operative risk, is the preferred option, while one of the two-staged procedures (primary-first or liver-first) should be pursued for the remaining patients. A recent network meta-analysis confirmed that no strategy to resect synchronous CRLM has superiority over the others in terms of survival, even if the liver-first approach was ranked as the best treatment for its relative efficacy based on 5-year OS outcomes. ${ }^{19}$ This evidence is too weak to propose the reverse strategy as standard.

All studies that compared strategies for synchronous CRLM suffered from major heterogeneity among groups. Different approaches are scheduled for different patients. In the literature, ${ }^{17-19}$ as in the present series, patients undergoing a reverse approach have more advanced liver disease and more rectal tumors. To face this scenario, we decided to not only adopt a propensity score match to make populations comparable but to also stratify patients according to their hepatic tumor burden. We hypothesized that the surgical strategy may have a different impact on prognosis according to the severity of the disease.

Our hypothesis was confirmed. The liver-first approach had results similar to the other approaches in patients with unilobar synchronous CRLM but was associated with a clear survival advantage over both the primary-first and simultaneous approaches in patients with multiple bilobar metastases. Its superiority was confirmed on multivariable analysis on the whole series and after strict propensity score matching. Three-year survival rates after reverse strategy exceeded $65 \%$ and ranged between 50 and $60 \%$ in the other groups. These results are even more relevant if we consider that the primary-first group included only patients who completed the two resections, but not those who dropped out. Intention-to-treat analysis is expected to show an even wider difference in favor of the liver-first approach. Factors contributing to these results are still to be investigated, but some hypotheses can be advanced. First, reverse strategy prioritizes the treatment of the tumor site that is judged the most prognostically relevant, i.e. the liver. Second, the liver-first approach requires early management of patients by an expert multidisciplinary liver team. Some studies demonstrated that such management is associated with longer survival. ${ }^{35-37}$ Third, the reverse strategy maximizes the effectiveness of neoadjuvant chemotherapy because liver surgery is performed with optimal timing, without any treatment interruption, and at the peak of the response. Any delay in surgery after chemotherapy may lead to early tumor reactivation and poor prognosis. ${ }^{38,39}$ Fourth, in the primary-first approach, colorectal resection is associated with an immunological alteration that could favor liver metastases proliferation. ${ }^{40}$ Finally, in comparison with simultaneous resections, the liver-first approach minimized postoperative morbidity that may negatively impact prognosis. ${ }^{41}$

The main strength of this study was its clinical relevance. We proposed a tumor burden-driven strategy for patients with synchronous CRLM as candidates for a single-stage hepatectomy (Fig. 4). According to our results, the liver-first approach should be the preferred option for patients with multiple bilobar metastases, while simultaneous resection should be the preferred option for patients with solitary metastasis requiring a minor hepatectomy, even if the definition of 'minor hepatectomies' is debated. ${ }^{42}$ In the remaining patients, the staged procedures were equivalent, while simultaneous resections should be cautiously considered because they have increased mortality risk whenever a major hepatectomy is needed. We did not analyze patients undergoing a two-stage hepatectomy, but, according to the favorable results that we observed in
FIG. 4 Treatment strategy of synchronous colorectal liver metastases according to hepatic tumor burden and scheduled hepatectomy. CRLM colorectal liver metastases

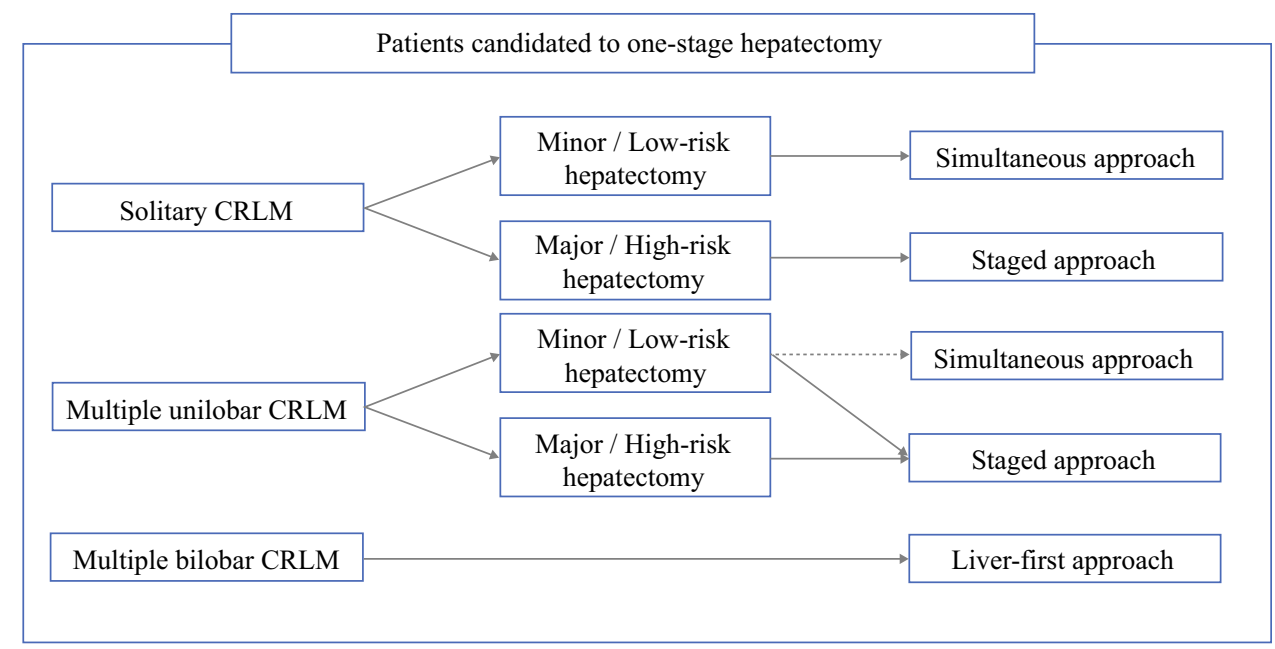


patients with simultaneous colorectal resection and unilobar limited resections, we can assume that the first stage of a two-stage hepatectomy can be safely combined with colorectal resection. Some limitations could be argued. This was a retrospective non-intention-to-treat analysis and the three groups had major heterogeneity at baseline. However, we collected a large number of patients from several centers worldwide and performed an accurate propensity score matching to make groups comparable, even if it led to a major reduction in sample size. Some prognostic data, such as CEA or RAS mutational status, were not considered and should be the subject of further analyses.

\section{CONCLUSION}

The surgical strategy in patients with colorectal cancer and synchronous liver metastases should be decided according to the hepatic tumor burden. In patients with multiple bilobar CRLM, we strongly suggest the liver-first approach as the standard because it is associated with excellent short-term results and longer survival than the alternative approaches.

\section{DISCLOSURE}

Santiago López-Ben has received grants from Olympus, Baxter and Integra, and Rene Adam has received honoraria for participation at meetings or congresses organized by Sanofi or Merck Serono. Felice Giuliante, Luca Viganò, Agostino M. De Rose, Darius F. Mirza, Réal Lapointe, Gernot Kaiser, Eduardo Barroso, Alessandro Ferrero, Helena Isoniemi, Irinel Popescu, Jean-Francois Ouellet, Catherine Hubert, Jean-Marc Regimbeau, Jen-Kou Lin, Oleg G. Skipenko, and Francesco Ardito have no conflicts of interest to declare.

Supplementary InformationThe online version contains supplementary material available at https://doi.org/10.1245/s10434021-10220-w.

ACKNOWLEDGEMENT The authors thank Aurélia Meurisse and all contributing centers for collecting the data.

OPEN ACCESS This article is licensed under a Creative Commons Attribution 4.0 International License, which permits use, sharing, adaptation, distribution and reproduction in any medium or format, as long as you give appropriate credit to the original author(s) and the source, provide a link to the Creative Commons licence, and indicate if changes were made. The images or other third party material in this article are included in the article's Creative Commons licence, unless indicated otherwise in a credit line to the material. If material is not included in the article's Creative Commons licence and your intended use is not permitted by statutory regulation or exceeds the permitted use, you will need to obtain permission directly from the copyright holder. To view a copy of this licence, visit http://creativecommons. org/licenses/by/4.0/.

FUNDING Open access funding provided by Università Cattolica del Sacro Cuore within the CRUI-CARE Agreement. No financial support was received for this study

\section{REFERENCES}

1. Ferlay J, Colombet M, Soerjomataram I, et al. Cancer incidence and mortality patterns in Europe: estimates for 40 countries and 25 major cancers in 2018. Eur J Cancer. 2018;103:356-87.

2. The International Agency for Research on Cancer (IARC): New Global Cancer Data: GLOBOCAN 2018. https://www.uicc.org/ new-global-cancer-data-globocan-2018\#

3. Fong Y, Fortner J, Sun RL, et al. Clinical score for predicting recurrence after hepatic resection for metastatic colorectal cancer: analysis of 1001 consecutive cases. Ann Surg. 1999;230:309-18.

4. Simmonds PC, Primrose JN, Colquitt JL, et al. Surgical resection of hepatic metastases from colorectal cancer: a systematic review of published studies. Br J Cancer. 2006;94:982-99.

5. Viganò L, Ferrero A, Lo Tesoriere R, et al. Liver surgery for colorectal metastases: results after 10 years of follow-up. Longterm survivors, late recurrences, and prognostic role of morbidity. Ann Surg Oncol. 2008;15:2458-64.

6. Adam R, de Gramont A, Figueras J, et al. Managing synchronous liver metastases from colorectal cancer: a multidisciplinary international consensus. Cancer Treat Rev. 2015;41:729-41.

7. Van Cutsem E, Cervantes A, Adam R, et al. ESMO consensus guidelines for the management of patients with metastatic colorectal cancer. Ann Oncol. 2016;27:1386-422.

8. Viganò L, Russolillo N, Ferrero A, et al. Evolution of long-term outcome of liver resection for colorectal metastases: analysis of actual 5-year survival rates over two decades. Ann Surg Oncol. 2012;19:2035-44.

9. Ardito F, Vellone M, Cassano A, et al. Chance of cure following liver resection for initially unresectable colorectal metastases: analysis of actual 5-year survival. $J$ Gastrointest Surg. 2013;17:352-9.

10. Creasy JM, Sadot E, Koerkamp BG, et al. Actual 10-year survival after hepatic resection of colorectal liver metastases: what factors preclude cure? Surgery. 2018;163:1238-44.

11. Mentha G, Majno PE, Andres A, et al. Neoadjuvant chemotherapy and resection of advanced synchronous liver metastases before treatment of the colorectal primary. $\mathrm{Br} J$ Surg. 2006;93:872-8.

12. Brouquet A, Mortenson MM, Vauthey JN, et al. Surgical strategies for synchronous colorectal liver metastases in 156 consecutive patients: classic, combined or reverse strategy? J Am Coll Surg. 2010;210:934-41.

13. van der Pool AE, de Wilt JH, Lalmahomed ZS, et al. Optimizing the outcome of surgery in patients with rectal cancer and synchronous liver metastases. Br J Surg. 2010;97:383-90. 
14. Viganò L, Karoui M, Ferrero A, et al. Locally advanced mid/low rectal cancer with synchronous liver metastases. World J Surg. 2011;35:2788-95.

15. Andres A, Toso C, Adam R, et al. A survival analysis of the liverfirst reversed management of advanced simultaneous colorectal liver metastases: a LiverMetSurvey-based study. Ann Surg. 2012;256:772-8.

16. Mayo SC, Pulitano C, Marques H, et al. Surgical management of patients with synchronous colorectal liver metastasis: a multicenter international analysis. J Am Coll Surg. 2013;216:707-16.

17. Esposito F, Lim C, Sa Cunha A, et al. Primary tumor versus liverfirst approach for synchronous colorectal liver metastases: an association Française de Chirurgie (AFC) multicenter-based study with propensity score analysis. World $J$ Surg. 2018;42:4046-53.

18. Valdimarsson VT, Syk I, Lindell G, et al. Outcomes of liver-first strategy and classical strategy for synchronous colorectal liver metastases in Sweden. HPB (Oxford). 2018;20:441-7.

19. Gavriilidis P, Katsanos K, Sutcliffe RP, et al. Simultaneous, delayed and liver-first hepatic resections for synchronous colorectal liver metastases: a systematic review and network metaanalysis. J Clin Med Res. 2019;11:572-82.

20. Magouliotis DE, Tzovaras G, Diamantis A, et al. A meta-analysis of liver-first versus classical strategy for synchronous colorectal liver metastases. Int J Colorectal Dis. 2020;35:537-46.

21. Raoux L, Maulat C, Suc B, et al. Impact of the strategy for curative treatment of synchronous colorectal cancer liver metastases. J Visc Surg. 2020;157(4):289-99.

22. Welsh FK, Chandrakumaran K, John TG, et al. Propensity scorematched outcomes analysis of the liver-first approach for synchronous colorectal liver metastases. Br J Surg. 2016;103:600-6.

23. Viganò L, Capussotti L, Lapointe R, et al. Early recurrence after liver resection for colorectal metastases: risk factors, prognosis, and treatment. A LiverMetSurvey-based study of 6025 patients. Ann Surg Oncol. 2014;21:1276-86.

24. Viganò L, Capussotti L, Barroso E, et al. Progression while receiving preoperative chemotherapy should not be an absolute contraindication to liver resection for colorectal metastases. Ann Surg Oncol. 2012;19:2786-96.

25. Allard MA, Adam R, Giuliante F, et al. Long-term outcomes of patients with 10 or more colorectal liver metastases. Br J Cancer. 2017;117:604-11.

26. Vallance AE, van der Meulen J, Kuryba A, et al. The timing of liver resection in patients with colorectal cancer and synchronous liver metastases: a population-based study of current practice and survival. Colorectal Dis. 2018;20:486-95.

27. Capussotti L, Ferrero A, Viganò L, et al. Major liver resections synchronous with colorectal surgery. Ann Surg Oncol. 2007; 14:195-201.

28. Hillings $\varnothing$ JG, Wille-Jørgensen P. Staged or simultaneous resection of synchronous liver metastases from colorectal cancer: a systematic review. Colorectal Dis. 2009;11:3-10.

29. Yoshioka R, Hasegawa K, Mise Y, et al. Evaluation of the safety and efficacy of simultaneous resection of primary colorectal cancer and synchronous colorectal liver metastases. Surgery. 2014;155:478-85.
30. Shubert CR, Habermann EB, Bergquist JR, et al. A NSQIP review of major morbidity and mortality of synchronous liver resection for colorectal metastasis stratified by extent of liver resection and type of colorectal resection. J Gastrointest Surg. 2015;19:1982-1294.

31. Idrees JJ, Bagante F, Gani F, et al. Population level outcomes and costs of single stage colon and liver resection versus conventional two-stage approach for the resection of metastatic colorectal cancer. HPB (Oxford). 2019;21:456-64.

32. Snyder RA, Hao S, Irish W, et al. Thirty-day morbidity after simultaneous resection of colorectal cancer and colorectal liver metastasis: American College of Surgeons NSQIP analysis. J Am Coll Surg. 2020;230:617-27.

33. Sturesson C, Valdimarsson VT, Blomstrand E, et al. Liver-first strategy for synchronous colorectal liver metastases-an intention-to-treat analysis. HPB (Oxford). 2017;19:52-8.

34. Ayez N, Burger JW, van der Pool AE, et al. Long-term results of the "liver first" approach in patients with locally advanced rectal cancer and synchronous liver metastases. Dis Colon Rectum. 2013;56:281-7.

35. Vallance AE, vanderMeulen J, Kuryba A, et al. Impact of hepatobiliary service centralization on treatment and outcomes in patients with colorectal cancer and liver metastases. Br J Surg. 2017;104:918-25.

36. Fenton HM, Taylor JC, Lodge JPA, et al. Variation in the use of resection for colorectal cancer liver metastases. Ann Surg. 2019;270:892-8.

37. Viganò L, Langella S, Ferrero A, et al. Colorectal cancer with synchronous resectable liver metastases: monocentric management in a hepatobiliary referral center improves survival outcomes. Ann Surg Oncol. 2013;20:938-45.

38. Vigano L, Darwish SS, Rimassa L, et al. Progression of colorectal liver metastases from the end of chemotherapy to resection: a new contraindication to surgery? Ann Surg Oncol. 2018;25:1676-85.

39. Mentha G, Terraz S, Morel P, et al. Dangerous halo after neoadjuvant chemotherapy and two-step hepatectomy for colorectal liver metastases. Br J Surg. 2009;96:95-103.

40. Shakhar G, Ben-Eliyahu S. Potential prophylactic measures against postoperative immunosuppression: could they reduce recurrence rates in oncological patients? Ann Surg Oncol. 2003;10:972-92.

41. Dorcaratto D, Mazzinari G, Fernandez M, et al. Impact of postoperative complications on survival and recurrence after resection of colorectal liver metastases: systematic review and meta-analysis. Ann Surg. 2019;270:1018-27.

42. Viganò L, Torzilli G, Troisi R, et al. Minor hepatectomies: focusing a blurred picture: analysis of the outcome of 4471 open resections in patients without cirrhosis. Ann Surg. 2019;270:842-51.

Publisher's Note Springer Nature remains neutral with regard to jurisdictional claims in published maps and institutional affiliations. 